Gefässchirurgie 2016 $\cdot 21: 440-446$ DOI 10.1007/s00772-016-0190-2

(C) Springer-Verlag Berlin Heidelberg 2016

CrossMark

H. Görtz' · J. Teßarek' · P. Berg' • I. Flessenkämper ${ }^{3} \cdot$ R. Ghotbi ${ }^{4}$ M. Hofmann ${ }^{5}$.

P. Kasprzak $^{6} \cdot$ R. Kellersmann ${ }^{7} \cdot$ V. Reichert ${ }^{8} \cdot$ A. Stehr ${ }^{9} \cdot$ G. Straeten ${ }^{2} \cdot$ T. Umscheid $^{10}$. C. Wack ${ }^{11} \cdot$ G. Torsello ${ }^{12}$

'Abteilung für Gefäßchirurgie, Bonifatius Hospital Lingen, Lingen, Deutschland

${ }^{2}$ Marienhospital Kevelaer, Kevelaer, Deutschland

${ }^{3}$ Berlin, Deutschland

${ }^{4}$ Abteilung für Gefäßchirurgie, Helios Klinikum München-West, München, Deutschland

${ }^{5}$ Schön Klinik Vogtareuth, Vogtareuth, Deutschland

${ }^{6}$ Universitätsklinikum Regensburg, Regensburg, Deutschland

${ }^{7}$ Universitätsklinikum Würzburg, Würzburg, Deutschland

${ }^{8}$ Klinikum Sindelfingen, Sindelfingen, Deutschland

${ }^{9}$ Evangelisches Krankenhaus Mülheim/Ruhr, Mülheim/Ruhr, Deutschland

${ }^{10}$ Helios Klinikum Bad Schwalbach, Bad Schwalbach, Deutschland

${ }^{1}$ Rotkreuzklinikum München, München, Deutschland

${ }^{12}$ Abteilung für Gefäßchirurgie, St. Franziskus-Hospital Münster, Münster, Deutschland

\title{
Novellierung des endovaskulären Curriculums zum 1.1.2017
}

\section{Was wird anders?}

Der Facharzt für Gefäßchirurgie ist in der Weiterbildungsordnung (WBO) der Ärztekammern verankert. Die endovaskulären Techniken sind bereits in der aktuellen Fassung fester Bestandteil dieser WBO. Art und Umfang der geforderten Weiterbildung befinden sich jedoch aufgrund der rasanten Entwicklung endovaskulärer Verfahren nicht mehr auf dem heute praktizierten und etablierten Niveau moderner Gefäßtherapie.

Um diesem Sachverhalt Rechnung zu tragen, war durch die Sektion für endovaskuläre Techniken der DGG bereits 2007 ein Curriculum mit einem begleitenden Kurssystem entwickelt worden, welches Gefäßchirurgen mit Facharztstatus ermöglichen sollte, ihre Kenntnisse in diesem Bereich der Gefäßmedizin zu vertiefen [1]. Der Nachweis der Kursteilnahme sowie die Vorlage einer im Curriculum geforderten Anzahl von selbstständig durchgeführten Eingriffen mündeten in die Zertifizierung zum ,Endovaskulären Chirurgen“ als Nachweis einer breit gefächerten endovaskulären Weiterbildung und zum „Endovaskulären Spezialisten“ als eine darüber hinausgehende Qualifikation zur eigenstän- digen Durchführung komplexer endovaskulärer Prozeduren.

Diese Zertifikate haben seit 2007 den Prozess einer Markenbildung durchlaufen und sich als feststehende Größe etabliert. Einige Ärztekammern haben bei der Vergabe von Weiterbildungsermächtigungen Wert auf deren Nachweis gelegt. Auch bei forensischen Fragestellungen, z. B. in Bezug auf die Qualifikation von Operateuren, war der Nachweis von großer Wichtigkeit. Die Begriffe „Endovaskulärer Chirurg“ und „Endovaskulärer Spezialist" haben sich somit als Marken etabliert. Vermutlich nicht zuletzt aufgrund der Anerkennung dieser Zertifikate über die Fachgesellschaft hinaus haben Kardiologen und Angiologen ein eigenes Fortbildungskonzept im Jahre 2012 vorgelegt [2].

Die rasante Weiterentwicklung der endovaskulären Techniken auf dem arteriellen wie venösen Sektor hat eine Aktualisierung des Curriculums notwendig gemacht. Zudem soll die Vermittlung der aktualisierten Lerninhalte mit einem Mehr an didaktischer Kompetenz erfolgen. Nach entsprechendem Abstimmungsprozess zwischen dem Vorstand der DGG und der Sektion für endovaskuläre Techniken möchten wir Ihnen im Folgenden das neue Konzept vorstellen, welches die bisherige Regelung ab 1.1.2017 ablöst.

\section{Allgemeines}

Ähnlich wie die in Erarbeitung befindliche Novelle der Weiterbildungsordnung ist auch das neue Curriculum kompetenzbasiert. Es sind insgesamt 3 Kompetenzlevel zu durchlaufen.

Im Level 1 geht es um den Erwerb von allgemeinen und organbezogenen Kenntnissen. Dieser Level wird durch den Besuch angebotener Kurse absolviert. Die Inhalte für die Kurse sind in diesem Curriculum festgeschrieben. Jeder Kursveranstalter wird zudem optional, ab 2018 verbindlich, für seine Kurse einen Syllabus erstellen, dem neben den Lernzielen auch die angewandten Lernmethoden und die kursbezogenen Literaturangaben zum Selbststudium zu entnehmen sind. Schwerpunkt der Kurse soll weiterhin die Vermittlung praktischer Fähigkeiten sein. Es werden daher immer auch Übungen am Simulator, 


\begin{tabular}{|ll|}
\hline Kompetenzblock & Kompetenzlevel 1 \\
\hline & Allgemeine und organbezogene Kenntnisse \\
\hline $\begin{array}{l}\text { Grundkenntnisse } \\
\text { endovaskulären } \\
\text { Arbeitens }\end{array}$ & - Röntgenbildgebung: neben CT und MR ins- \\
& graphie, Roadmapverfahren, Bildbearbeitung, \\
& CO2-Angiographie \\
- & Indikationen und Kontraindikationen \\
- & Kontrastmitteleigenschaften, Nebenwirkungen \\
& von KM (CIN), Therapie von Kontrastmittelreaktio- \\
& nen \\
- & Strahlenschutz: Einflüsse auf die Strahlendosis; \\
& Methoden zur Reduktion: apparativer Strahlen- \\
& schutz und Abschirmung; Strahlengeometrie; ALA- \\
& RA-Regel; Richtwerte BfS; gesetzliche Grundlagen; \\
& ärztliche Stelle \\
- & Punktionstechniken mit und ohne Ultraschall; \\
& Drähte und Schleusen; Struktur und Funktion ver- \\
& schiedener Schleusen; Ballons; Mikro-/Katheter; \\
& Coils und Plugs; Verschlusssysteme; pharmakologi- \\
& sches perioperatives Management \\
- & Spezifische Kenntnisse über die unterschiedlichen \\
& Zulassungen von Implantaten
\end{tabular}

Kompetenzlevel 2

Assistierte technische Durchführung

- Punktionen, Angiographien und Interventionen mit Ballon und Stent sowie Endografts unter besonderer Berücksichtigung des Strahlenschutzes unter Einhaltung der Dosisrichtwerte, im Besonderen bei unterschiedlicher C-Armposition (z. B. transbrachialer Zugang, retrograder Zugang am Unterschenkel)

- Verschlusssysteme (Anker und Nahtsysteme)

- Kenntnisse über das endovaskuläre und operative Komplikationsmanagement von Fehlpunktionen und Fehlplatzierung von Verschlusssystemen

- Differenzierte Kenntnisse über unterschiedliche Stents (BMS, covered Stents, DES), deren Besonderheiten und Indikationen sowie die auf das Gefäßsegment bezogene Datenlage

- Grundsätzliche Kenntnisse über mechanische Verfahren zur Wiedereröffnung von akut und chronisch verschlossenen Arterien und Venen (CTO-Geräte, Rotations- und Aspirationsthrombektomie)

\begin{tabular}{|c|c|c|}
\hline $\begin{array}{l}\text { pAVK Aorta und } \\
\text { Becken }\end{array}$ & $\begin{array}{l}\text { - Indikation zu PTA/Stenting der Beckenetage, Ma- } \\
\text { terialauswahl, Einsatz von großlumigen ungeco- } \\
\text { verten/gecoverten Stents bei Läsionen der Aorta } \\
\text { - Zugänge zum Becken retrograd, simultan bds., } \\
\text { cross-over/transbrachialer Zugang mit/ohne lange } \\
\text { Schleuse } \\
\text { - Therapie von Läsionen der A. iliaca interna } \\
\text { - Rekanalisationstechniken von CTO (retrograd, }\end{array}$ & $\begin{array}{l}\text { - PTA/Stenting isolierter Aorten/ } \\
\text { Beckenläsionen, retrograd und cross- } \\
\text { over } \\
\text { - Behandlung komplexer Läsionen; CTO; } \\
\text { Verwendung von Schutzdraht; Protekti- } \\
\text { on der A. iliaca interna durch Ballonok- } \\
\text { klusion bei Iliakalgabelläsionen } \\
\text { - Rezidiveingriffe bei In-Stent-Restenosen }\end{array}$ \\
\hline
\end{tabular}

Kompetenzlevel $3^{\text {a }}$

Selbstständige Durchführung

- Selbstständige Durchführung von $50 \mathrm{Be}$ gründungen der rechtfertigenden Indikation

- 50 Punktionen mit und ohne Ultraschall

- $50 \times$ Gebrauch eines Verschlusssystems

- 50 Angiographien einschließlich anschließender, eigenständiger Bildbearbeitung, Dokumentation der Befunde

- Selbstständige Durchführung von $50 \mathrm{ln}$ terventionen an der Aorta/Beckenetage perkutan oder als $\mathrm{Hy}$ brideingriffe

\begin{tabular}{|c|c|c|c|}
\hline $\begin{array}{l}\text { pAVK } \\
\text { Oberschenkel }\end{array}$ & $\begin{array}{l}\text { - Kenntnisse zur Indikationsstellung von Interven- } \\
\text { tionen in Abhängigkeit der Offenheitsraten } \\
\text { - Zugang (antegrad, cross-over, retrograd, popliteal) } \\
\text { - Indikation für PTA/BMS/DEB/DES, gecoverte Stents } \\
\text { - Rekanalisationstechniken, Materialien, Vorgehen } \\
\text { bei Rezidivstenose, Verschluss } \\
\text { - Vorgehen bei kalzifizierter Läsion, Dissektion, } \\
\text { Kenntnisse zum Einsatz von mechanischen Throm- } \\
\text { bektomien/Atherektomie-Systemen } \\
\text { - Einsatz von Lyse-/Aspirationssystemen } \\
\text { - Indikationen zur Intervention an der A. Profunda }\end{array}$ & $\begin{array}{l}\text { - Interventionen der Oberschenkeletage } \\
\text { wie z. B. PTA, Stenting, Interventionen } \\
\text { bei Verschluss und bei komplexen Läsio- } \\
\text { nen; Einsatz von DEB } \\
\text { - Durchführung von Rotationsthrombek- } \\
\text { tomieverfahren und Atherektomie } \\
\text { - Behandlung von Rezidivläsionen und } \\
\text { Serviceinterventionen nach chirurgi- } \\
\text { schen Verfahren } \\
\text { - Hybridverfahren }\end{array}$ & $\begin{array}{l}\text { - Selbstständige Durch- } \\
\text { führung von } 50 \text { Inter- } \\
\text { ventionen perkutan } \\
\text { oder als Hybridverfah- } \\
\text { ren am Oberschenkel }\end{array}$ \\
\hline $\begin{array}{l}\text { Bauchaorten- } \\
\text { aneurysma (BAA) }\end{array}$ & $\begin{array}{l}\text { - Röntgenanatomie der Aorta und der Becken- } \\
\text { gefäße sowie ihrer Nebenäste bei CT-, MR- und } \\
\text { Katheterangiographie } \\
\text { - Einstellungstechniken (Angulation und Kippung) } \\
\text { - Prothesentypen, Sondierungskatheter, Drähte, } \\
\text { Zugangswege, Verschlusssysteme } \\
\text { - Spezielle Indikationen nach IFU } \\
\text { - Methodenbedingte Limitierungen und Komplika- } \\
\text { tionen der einzelnen Verfahren }\end{array}$ & $\begin{array}{l}\text { - Implantationen bei infrarenalem BAA } \\
\text { - Differenzierte Kenntnisse über das Kom- } \\
\text { plikationsmanagement } \\
\text { - Kenntnisse über therapeutische Mög- } \\
\text { lichkeiten bei Notfallpatienten sowie bei } \\
\text { schwierigen Anatomien; f-EVAR, b-EVAR, } \\
\text { ch-EVAR, lliac-side-branch-Prothese; } \\
\text { alternative endovaskuläre Behandlungs- } \\
\text { verfahren wie „coiling“ } \\
\text { - Prä-, peri- und postoperative Behand- } \\
\text { lung sowie Nachsorge }\end{array}$ & $\begin{array}{l}\text { - Selbstständige } \\
\text { Durchführung von } \\
20 \text { EVAR-Implantatio- } \\
\text { nen }\end{array}$ \\
\hline
\end{tabular}

${ }^{a}$ In den einzelnen Kompetenzblöcken werden Richtzahlen angegeben, die zur Erlangung der Eigenständigkeit in Bezug auf das endovaskuläre Arbeiten als sinnvoll angesehen werden.

BFS Bundesamt für Strahlenschutz; BMS "Bare Metal Stent"; CIN Contrastmittel Induzierte Nephropathie; CTO "Chronic Total Occlusion"; IFU „Instructions for Use"; KM Kontrastmittel; DEB "Drug Eluting Balloon"; DES "Drug Eluting Stent" 


\section{Editorial}

Tab. 2 Zertifikat „endovaskulärer Spezialist"

\begin{tabular}{|c|c|}
\hline \multicolumn{2}{|c|}{ KompetenzblockKompetenzlevel 1} \\
\hline & Allgemeine und organbezogene Kenntnisse \\
\hline $\begin{array}{l}\text { Karotis und } \\
\text { obere Extremi- } \\
\text { täten }\end{array}$ & $\begin{array}{l}\text { - Röntgenanatomie der intrazerebralen, supraaortalen } \\
\text { Gefäße und des Aortenbogens in der CT-, MR- und Ka- } \\
\text { theterangiographie; Einstellung des C-Bogens (a.-p., } 30^{\circ} \\
\text { LAO Aortenbogen, kaudokraniale Kippung) } \\
\text { - Identifikation geeigneter Anatomien, Indikationen, Kon- } \\
\text { traindikationen und Komplikationsmöglichkeiten, von } \\
\text { Stenttypen und Protektionssystemen (Filter, Okklusions- } \\
\text { system, „no flow", retrograder "flow", zervikaler Zugang) } \\
\text { - Pharmakologische Begleit- und Notfalltherapie }\end{array}$ \\
\hline \multirow{4}{*}{$\begin{array}{l}\text { Thorakales } \\
\text { Aortenaneu- } \\
\text { rysma, } \\
\text { Aorten- } \\
\text { dissektion }\end{array}$} & $\begin{array}{l}\text { Röntgenanatomie: } \\
\text { - Supraaortale Äste mit anatomischen Varianten } \\
\text { - Aortenbogenkonfigurationen } \\
\text { - Spinale Versorgung }\end{array}$ \\
\hline & $\begin{array}{l}\text { Pathologien des Aortenbogens und der thorakalen Aorta } \\
\text { sowie sich der daraus ergebenden therapeutischen Konse- } \\
\text { quenzen (z.B.): } \\
\text { - Traumatische Aortenruptur } \\
\text { - Dissektion } \\
\text { - Aneurysma } \\
\text { - Coarctatio } \\
\text { - IMH } \\
\text { - PAU }\end{array}$ \\
\hline & $\begin{array}{l}\text { Materialkunde und Einsatz der Endoprothesen (z.B.): } \\
\text { - Bestimmung der Landungszonen und Sizing der Prothe- } \\
\text { sen } \\
\text { - Methodenbedingte Limitationen für endovaskuläre } \\
\text { Standardverfahren }\end{array}$ \\
\hline & $\begin{array}{l}\text { Perioperatives Management einschließlich der Strategien } \\
\text { zur Vermeidung einer spinalen Ischämie (Neuromonitoring }\end{array}$ \\
\hline Viszeralgefäße & $\begin{array}{l}\text { - Röntgenanatomie der viszeralen Gefäße, der Niere und } \\
\text { der Gefäße des Beckens (inkl. Lumbalarterien in der CT-, } \\
\text { MR- und Katheterangiographie) } \\
\text { - Indikationen, Kontraindikationen und Komplikations- } \\
\text { möglichkeiten } \\
\text { - Identifikation geeigneter Anatomien, Krankheitsbilder } \\
\text { - Notwendige präinterventionelle Diagnostik } \\
\text { - Spezielle Materialkunde } \\
\text { - Einstellungstechniken der Röntgenanlage (a.-p., seitlich } \\
90 \text { Grad, Limitationen von Tisch und C-Bogen); Einstel- } \\
\text { lung des Gerätes bei Sondierung vom Arm } \\
\text { - Prä-, peri- und postoperatives Management }\end{array}$ \\
\hline
\end{tabular}

Hämodialyse- Verschiedene Dialysezugangsmodalitäten (a.v.-Fistel, Proshunts thesenshunt, modifizierte Prothesen) und ihre hämodynamischen Eigenschaften

- Grundkenntnisse über Flusseigenschaften von a.v.-Shunts und ihre Veränderungen bei gestörter Shuntfunktion (z. B. Shunt-induzierte Handischämie, Shuntdysfunktion Non-Maturation, Rezirkulation)

- Punktionstechniken zur angiographischen Shuntdarstellung

- Grundlagen zum Ablauf und zur Interpretation einer angiographischen Shuntdarstellung

- Grundkenntnisse über alternative Methoden (z. B. $\mathrm{CO}_{2}$-Angiographie, Phlebo-MR)

- Grundlagen verschiedener technischer Möglichkeiten von Dilatationsballons (z. B. Cutting-, Scoring-Ballon)

- Technische Möglichkeiten der endovaskulären Shuntthrombektomie (z. B. Fibrinolyse, mechanische Thrombektomiesysteme)

Kompetenzlevel 2

Assistierte technische Durchführung

- Endovaskuläre Prozeduren im supraaortalen Gefäßsegment (PTA, Stent, Coiling, exkl. intrakranielle Verfahren)

- Zusätzlich Kenntnisse über das Komplikationsmanagement, von Gefäßverschlüssen, peripheren Embolisationen und Dissektionen (Thrombolysetherapie und Aspirationsverfahren)

Endovaskuläre Implantationen von thora-

kalen Endoprothesen bei:

- Aneurysma

- Dissektion

- Traumatischer Aortenruptur

Zusätzlich Kenntnisse über therapeutische Möglichkeiten bei schwierigen Anatomien, insbesondere:

- Problematische Zugangswege

- Anatomische Varianten, wie z. B. A. lusoria, Vertebralarterien-Anomalien und Indikationen zur Erhaltung der Perfusion der A. subclavia

- Möglichkeiten der Verlängerung der Landungszone (Transpositionen, Debranching, Chimney, Seitenarmtechnologie)

Differenzierte Kenntnisse bezüglich des Komplikationsmanagements

Postoperative Behandlung und Follow-up

- Endovaskuläre Prozeduren in den viszeralen Gefäßen (PTA, Stent, Coiling)

- Kenntnisse über das Komplikationsmanagement, bei Dissektionen, Verschlüssen und peripheren Embolisationen (Thrombolysetherapie und Aspirationsverfahren)

- Sekundärinterventionen bei Viszeralgefäßverschluss nach fenestrierten Prothesen

- Handling bei fehlplatzierten Stents und Coils

- Dilatationen von Shuntstenosen (inklusive zentrale Stenosen) und kathetergestützte Thrombektomien

- Zusätzlich Kenntnisse über Indikationen für Stents und Stentgrafts. Anwendung von Hochdruck-, Cutting-, und Scoring-Ballons. Indikation zum Coiling von Seitenästen

- Endovaskuläre Möglichkeiten bei der Behandlung der Shunt-induzierten Handischämie (z. B. endovaskuläre DRAL)
Kompetenzlevel $3^{\mathrm{a}}$

Selbstständige

Durchführung

- Selbstständige Durchführung von 50 Eingriffen an den supraaortalen Gefäßen, davon 30 Eingriffe an der Arteria carotis (inklusive Hybrideingriffe)

- Selbstständige Durchführung von 10 thorakalen Stentimplantationen bei verschiedenen Indikationen

- Selbstständige Durchführung von 15 Eingriffen an den viszeralen Gefäßen

- Selbstständige Durchführung von 20 Interventionen am Dialyseshunt (auch im Hybridverfahren), davon 5 zentralvenöse Stenosen 


\begin{tabular}{|c|c|c|c|}
\hline \multicolumn{2}{|c|}{ KompetenzblockKompetenzlevel 1} & \multirow[b]{2}{*}{$\begin{array}{l}\text { Kompetenzlevel } 2 \\
\text { - Endovaskulären Prozeduren der Popli- } \\
\text { tealregion, (PTA, Rekanalisation, Läsions- } \\
\text { präparation und Stentimplantation bei } \\
\text { kalzifizierten Läsionen) } \\
\text { - Krurale PTA bei isolierten Läsionen, } \\
\text { Rekanalisation bei CTO } \\
\text { - Zusätzliche Kenntnisse über Korrektur- } \\
\text { möglichkeiten bei Drahtperforation und } \\
\text { Rekanalisation OSG übergreifend und } \\
\text { pedal } \\
\text { - Pedaler Zugang }\end{array}$} & \multirow{2}{*}{ 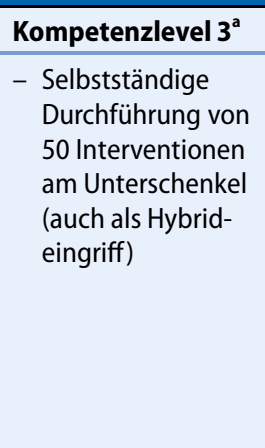 } \\
\hline $\begin{array}{l}\text { pAVK Poplitea } \\
\text { und Unter- } \\
\text { schenkel }\end{array}$ & $\begin{array}{l}\text { - Röntgenanatomie der popliteokruralen Gefäßregion und } \\
\text { ihrer häufigsten Varianten in der CT-, MR- und Katheter- } \\
\text { angiographie } \\
\text { - Indikationen nach aktueller Studienlage } \\
\text { - Lage/Bedeutung der Bewegungssegmente } \\
\text { - Kenntnis des Angiosom-Konzepts } \\
\text { - Befallsmuster pAVK } \\
\text { - Spezielle Materialkunde für die Interventionen am Unter- } \\
\text { schenkel } \\
\text { - Prä-, peri- und postinterventionelle medikamentöse } \\
\text { Therapie }\end{array}$ & & \\
\hline $\begin{array}{l}\text { Tiefes Venen- } \\
\text { system }\end{array}$ & $\begin{array}{l}\text { - Die Indikationsstellung zur Diagnostik und Erstellung } \\
\text { diagnostischer Phlebographien einschließlich der Erhe- } \\
\text { bung einer intraoperativen radiologischen Befundkon- } \\
\text { trolle } \\
\text { - Instrumentelle Untersuchungsverfahren einschließlich } \\
\text { der Durchblutungsmessung und den nicht invasiven } \\
\text { bildgebenden Verfahren sowie Erhebung eines angio- } \\
\text { logischen Befunds zur Operationsvorbereitung und } \\
\text { Nachsorge } \\
\text { - Kenntnisse zu doppler-/duplexsonographischen Unter- } \\
\text { suchungen der Extremitäten entsorgenden Gefäße } \\
\text { - Kenntnis und Durchführung phlebodynamischer Unter- } \\
\text { suchungen } \\
\text { - Spezielle Materialkunde } \\
\text { - Prä-, peri- und postoperatives Management }\end{array}$ & $\begin{array}{l}\text { - Rekanalisationen des tiefen Venensys- } \\
\text { tems, Thrombus beseitigende Katheter- } \\
\text { verfahren, Stenting, Fibrinolysebehand- } \\
\text { lung sowie Hybrideingriffe } \\
\text { - Die fachbezogene Beurteilung, Deutung } \\
\text { und Interpretation von phlebographi- } \\
\text { schen Darstellungen und Abbildungen } \\
\text { der Schnittbildtechniken (MRT, CT), } \\
\text { inklusive Durchführung von Therapie- } \\
\text { planungen für endovaskuläre Eingrif- } \\
\text { fe, Beurteilung und Ausmessung von } \\
\text { Schnittbilddiagnostiken }\end{array}$ & $\begin{array}{l}\text { - Selbstständige } \\
\text { Durchführung von } \\
20 \text { Eingriffen am } \\
\text { tiefen Venensystem }\end{array}$ \\
\hline $\begin{array}{l}\text { Besondere } \\
\text { Entitäten }\end{array}$ & Allgemeine und organbezogene Kenntnisse & Assistierte technische Durchführung & $\begin{array}{l}10 \text { Eingriffe selbst- } \\
\text { ständige Durchfüh- } \\
\text { rung aus a-d }\end{array}$ \\
\hline $\begin{array}{l}\text { a. Cava superior } \\
\text { und inferior }\end{array}$ & $\begin{array}{l}\text { - Röntgenanatomie der Hohlvenen } \\
\text { - Klinische Evaluation und Diagnostik } \\
\text { - Behandlung der Begleiterkrankungen (Gerinnungsstö- } \\
\text { rungen, Kathetersepsis etc.) } \\
\text { - Indikationen und Kontraindikationen für die Verfahren } \\
\text { - Rekanalisationstechniken (transfemoral, transbrachial, } \\
\text { Rendezvoustechnik), Stentindikationen } \\
\text { - Besonderheiten der Anatomie: Lebervenen, Vorhofana- } \\
\text { tomie, anatomische Varianten } \\
\text { - Spezielle Materialkunde, Prä-, peri- und postoperatives } \\
\text { Management }\end{array}$ & $\begin{array}{l}\text { Kathetergestütze Eingriffe an der V. ca- } \\
\text { va superior oder inferior (inklusive Kava- } \\
\text { schirm) }\end{array}$ & $\begin{array}{l}\text { Kathetergestütze } \\
\text { Eingriffe an der V. cava } \\
\text { superior oder inferior } \\
\text { (inklusive Kavaschirm) }\end{array}$ \\
\hline b. Gefäßtrauma & $\begin{array}{l}\text { - Röntgenanatomie der Gefäße in der CT-, MR- und Kathe- } \\
\text { terangiographie } \\
\text { - Kenntnis der Pathologie (direkte und indirekte, stumpfe } \\
\text { und scharfe Verletzungen) mit möglichen Folgeschäden } \\
\text { - Spezielle Materialkunde einschließlich aller Embolisati- } \\
\text { onstechniken, Aspirationsverfahren, Fibrinolyse } \\
\text { - Projektionsebenen der Röntgenanlage } \\
\text { - Identifikation geeigneter Anatomien zur endovaskulären } \\
\text { Behandlung bei Gefäßverletzungen (Bedeutung der } \\
\text { Bewegungssegmente) } \\
\text { - Prä-, peri- und postoperatives Management }\end{array}$ & $\begin{array}{l}\text { - Endovaskuläre Prozeduren in allen Ge- } \\
\text { fäßregionen (Stenting, Coiling). } \\
\text { - Zusätzlich Kenntnisse über das Kom- } \\
\text { plikationsmanagement in der Behand- } \\
\text { lung von Gefäßtraumata, (Dissektionen, } \\
\text { Verschlüssen und peripheren Emboli- } \\
\text { sationen sowie weiterer Blutung und } \\
\text { Gefäßrupturen. Schock, Reperfusions- } \\
\text { syndrom) }\end{array}$ & $\begin{array}{l}\text { - Endovaskuläre } \\
\text { Prozeduren in allen } \\
\text { Gefäßregionen } \\
\text { (Stenting, Coiling) }\end{array}$ \\
\hline $\begin{array}{l}\text { c. Lungenem- } \\
\text { bolie }\end{array}$ & $\begin{array}{l}\text { - Klinische Evaluation und Diagnostik, Wells-Score LE, } \\
\text { GENEVA-Score, Evaluation der Bildgebung } \\
\text { - Spezifische, gebietsbezogene Hämostaseologie } \\
\text { - Zugangswege zur endovenösen Therapie } \\
\text { - Aspirationsverfahren, Fragmentierung, Fibrinolyse } \\
\text { - Spezielle Materialkunde } \\
\text { - Prä-, peri- und postoperatives Management }\end{array}$ & $\begin{array}{l}\text { Kathetergestütze Thrombektomien und } \\
\text { Lysebehandlungen } \\
\text { - Kenntnisse über das Komplikationsma- } \\
\text { nagement, „,bail out“ (Sternotomie und } \\
\text { operative Thrombektomie) }\end{array}$ & $\begin{array}{l}\text { Kathetergestütze } \\
\text { Thrombektomien und } \\
\text { Lysebehandlungen }\end{array}$ \\
\hline
\end{tabular}


d. AV-Malformationen

- Pathophysiologie der verschiedenen (nicht intrakraniellen) Malformationen, spezielle Anatomie

- Diagnostische Maßnahmen

- Zugangstechniken arteriell wie venös

- Spezielle Materialkunde (Coils, Mikrocoils, Plugs, Sklerosierungsmittel)

- Kompatibilität der Instrumente und Implantate

- Prä-, peri- und postoperatives Management
- Kathetergestütze Behandlungen von AV-Malformationen

- Kenntnisse von Bail-out-Verfahren bei Verlust von Coils oder anderen Implantaten

- Gewebekleber und deren Begleitreaktionen
Kathetergestütze Behandlungen von AV-Malformationen

${ }^{a}$ In den einzelnen Kompetenzblöcken werden Richtzahlen angegeben, die zur Erlangung der Eigenständigkeit in Bezug auf das endovaskuläre Arbeiten als sinnvoll angesehen werden.

CTO „Chronic Total Occlusion“; DRAL "Distal Radial Artery Ligation“; IMH Intramurales Hämatom; PAU Penetrierendes Aortenulcus

„live cases“ oder Fallbesprechung mit Videodokumentation obligater Bestandteil eines Kurses sein.

Um die Qualität der Kurse zu gewährleisten, werden diese zuvor von der Privaten Akademie der DGG zertifiziert. Zudem sind die Kursleiter aufgefordert, bis 2018 einen Kurs zu absolvieren, der sich inhaltlich mit der didaktisch kompetenten Vermittlung von Wissen beschäftigt, sog. Train-the-trainer-Kurs. Ein solcher Kurs wird beispielsweise während des Konvents im Frühjahr 2017 angeboten. Es ist das Ziel der DGG, nicht nur inhaltlich, sondern auch pädagogisch ein hohes Niveau bei den Kursen bieten zu können. Ausgehend von den endovaskulären Kursen wird nach und nach das gesamte Kurskonzept der Privaten Akademie der DGG in dieser Form überarbeitet werden.

Beim Level 2 steht der Erwerb praktischer Erfahrungen durch Assistenzen sowie assistierte Prozeduren im Mittelpunkt. Ferner sollen aufgrund der gesammelten praktischen Erfahrungen Grundkenntnisse beim Komplikationsmanagement erworben bzw. vertieft werden. Ein spezieller Nachweis für die Absolvierung des 2. Levels ist nicht erforderlich.

Nach Durchlaufen dieses zweiten Levels soll im 3. Level nachgewiesen werden, dass die in Frage kommenden Eingriffe selbstständig durchführt werden können. Ähnlich wie in der Weiterbildungsordnung sind hier in den meisten Kategorien Richtzahlen hinterlegt. Das Erreichen dieser Richtzahl ist durch Vorlage eines Logbuches mit laufenden OPZiffern nachzuweisen. Diese Anzahl ist so bemessen, dass der Erwerber auch praktisch und nachvollziehbar zum Kompli- kationsmanagement befähigt sein soll. Eine Fokussierung auf nur wenige Kliniken, in denen man diese Kompetenzen erwerben kann, wurde bewusst vermieden.

Das neue Curriculum ist kompetenzbasiert. Es muss daher die erworbene Kompetenz vor Erteilung des Zertifikates nachgewiesen werden. Dieser Nachweis erfolgt durch eine Prüfung. Zu dieser Prüfung kann sich nur anmelden, wer

1. die erforderlichen Kurse absolviert hat,

2. die Fachkunde im Strahlenschutz für das Organgebiet Gefäße sowie die darauf aufbauende Fachkunde für Interventionen und

3. anhand des Logbuchs das Erreichen der Richtzahlen

nachweisen kann.

\section{》) Das neue Curriculum ist kompetenzbasiert}

Die Prüfungen haben einen theoretischen und einen praktischen Teil. Bei der Überprüfung der Theorie geht es um die Kenntnisse der aktuellen Datenlage und ggf. gültiger Leitlinien in den zu prüfenden Bereichen. Eine Publikationsanalyse, wie sie bei der europäischen Prüfung erforderlich ist, wird nicht erwartet. Zudem soll der Kandidat am Simulator nachweisen, dass er während seiner Ausbildung ausreichende handwerkliche Fähigkeiten erworben hat. Die Prüfungen werden an zentralen Veranstaltungen der DGG, z. B. während des Jahreskongresses, der Sommerakademie oder der Frühjahrstagung, regelmäßig angeboten.
Nach bestandener Prüfung erhält der Kandidat das Zertifikat „Endovaskulärer Chirurg“ oder „Endovaskulärer Spezialist".

\section{Begriffsdefinition nach neuem Curriculum}

\section{„Endovaskulärer Chirurg"}

Hierbei handelt es sich weiterhin um das Basiszertifikat. Es beinhaltet folgende Module, für die allesamt die Kompetenzen im Level 1 und 3 nachgewiesen werden müssen. Die einzelnen Inhalte und Richtzahlen sind festgelegt (• Tab. 1):

1. Grundkenntnisse endovaskulären Arbeitens.

2. Endovaskuläre Behandlung des infrarenalen Bauchaortenaneurysmas mittels Endoprothesen (EVAR).

3. Endovaskuläre Behandlung der Aorta und der Beckenetage bei pAVK.

4. Endovaskuläre Behandlung der Oberschenkeletage bei pAVK.

Nach Durchlaufen aller Kompetenzlevel kann der abschließende Nachweis durch die Prüfung erfolgen.

\section{„Endovaskulärer Spezialist"}

Für dieses weiterführende Zertifikat ist der vorherige Erwerb des Zertifikats „Endovaskulärer Chirurg“ Voraussetzung.

Insgesamt besteht dieses Zertifikat aus 7 unterschiedlichen Modulen.

Da nicht jeder alles vorhält, müssen 4 der 7 Module in allen 3 Kompetenzleveln durchlaufen werden. Auch hier sind die Inhalte und die Richtzahlen festgelegt. 
Folgende Module stehen zur Auswahl (- Tab. 2):

1. Endovaskuläre Behandlung der supraortalen und suprakardialen Gefäße.

2. Endovaskuläre Behandlung des thorakalen Aortenaneurysmas.

3. Endovaskuläre Behandlung der Viszeralgefäße einschließlich fenestrierter und gebranchter Endoprothesen.

4. Endovaskuläre Behandlung von Dialyseshunts.

5. Endovaskuläre Behandlung der Unterschenkeletage bei pAVK.

6. Endovaskuläre Behandlung der tiefen Venen.

7. Endovaskuläre Behandlung besonderer Entitäten:

a. Cava superior und inferior,

b. Trauma,

c. Lungenarterienembolie,

d. AV-Malformationen.

Auch hier steht am Ende vor Erteilung des Zertifikats eine Überprüfung. Die Prüfungsinhalte stammen ausschließlich aus den 4 Modulen, die vom Kandidaten gewählt worden sind.

\section{Weitere Neuerungen}

Aufgrund der schnellen Entwicklung der auf dem endovaskulären Sektor zur Verfügung stehenden Systeme, haben sich DGG-Vorstand und Sektion dahingehend geeinigt, die Laufzeit der Zertifikate auf 5 Jahre zu befristen. Die Frist wird automatisch verlängert, sobald der Inhaber des Zertifikats nachweisen kann, dass er im 5-Jahres-Zeitraum an einem von der Akademie ausgeschriebenen Auffrischungskurs (z. B. technisches Forum) teilgenommen hat.

\section{》) Die Laufzeit der Zertifikate ist auf 5 Jahre zu befristen}

Die Erteilung der Zertifikate ist an den Facharzt für Gefäßchirurgie gebunden, d. h. die bestandene Facharztprüfung ist Voraussetzung für die Erteilung des Zertifikats. Allerdings ist es sehr wohl möglich, bereits während der Weiterbildungszeit zum Facharzt mit dem Erwerb zu beginnen. Es ist also möglich, bereits Kurse zu absolvieren und Eingriffe selbstständig durchzuführen. Da sich die Richtzahlen auf einen Weiterbildungszeitraum von 6 Jahren (nach gültigen WBO) beziehen, erscheinen die Vorgaben durchaus erfüllbar.

\section{Übergangsregelungen}

Wiebei jeder Veränderung wurden Übergangsregelungen festgelegt. Diese betreffen zum einen den Erwerb, zum anderen die Gültigkeit der Zertifikate.

Alle diejenigen, die innerhalb eines halben Jahres nach Inkraftsetzung des neuen Curriculums, d.h. vor dem 30.6.2017, mit dem Erwerb des jeweiligen Zertifikats durch Teilnahme an einem Kurs begonnen haben, erhalten dies noch nach den Regelungen des alten Curriculums. Alle anderen müssen das neue Curriculum absolvieren. Wer zudem nach den alten Vorgaben die Zertifikate erhalten hat, ist im Sinne des Bestandsschutzes von der Befristungsregelung ausgeschlossen, auch wenn die Teilnahme an den vorgesehenen Auffrischungskursen empfohlen wird.

\section{Zusammenfassung}

Die DGG hat sich das Ziel gesetzt, die Fortbildung der Gefäßchirurgen zu unterstützen, um den steigenden Bedarf an endovaskulärer Kompetenz abzudecken. Diese Kompetenz muss nach außen auch durch die Zertifikate sichtbar sein. Durch Anwendung des von der Bundesärztekammer favorisierten Kompetenzmodells mit abschließender Überprüfung gewinnt das Zertifikat deutlich an Wert. Ebenfalls werden auch die Kursveranstalter durch Absolvierung von Trainingskursen und der Entwicklung eines Syllabus für ihre Kurse zu einer Steigerung der Wertigkeit beitragen. Dieses bedeutet zwar für alle einen Mehraufwand, der Gefäßchirurg der Zukunft aber muss unserer festen Überzeugung nach mit dem gesamten Spektrum operativer und endovaskulärer Techniken vertraut sein, denn nur so ist er in der Lage, eine umfassende Versorgung des Gefäßpatienten anzubieten. Dieses kann in den Ballungsgebieten in Zusammenarbeit mit den anderen
Fachdisziplinen realisiert werden. In der Flächenversorgung unseres Landes muss der Gefäßchirurg in der Lage sein, alle Therapieoptionen anzubieten, um die gefäßmedizinische Versorgung der Bevölkerung aufrecht $\mathrm{zu}$ erhalten.

\section{Korrespondenzadresse}

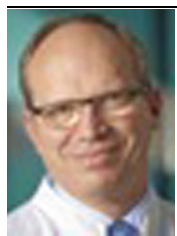

Dr. H. Görtz

Abteilung für Gefäßchirurgie, Bonifatius Hospital Lingen Wilhelmstr. 13, 49808 Lingen, Deutschland hartmut.goertz@hospitallingen.de

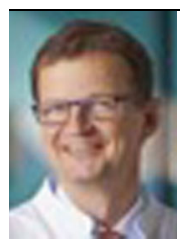

Dr. J. Teßarek

Abteilung für Gefäßchirurgie, Bonifatius Hospital Lingen Wilhelmstr. 13, 49808 Lingen, Deutschland joerg.tessarek@hospitallingen.de

Interessenkonflikt. H. Görtz, J. Teßarek, P. Berg, I. Flessenkämper, R. Gothbi, M. Hofmann, P. Kasprzak, R. Kellersmann, V. Reichert, A. Stehr, G. Straeten, T. Umscheid, C. Wack und G. Torsello geben an, dass kein Interessenkonflikt besteht.

\section{Literatur}

1. Flessenkämper I, Gussmann A, Berg $P$, Görtz $H_{\text {, }}$ Heider $P$, Heidrich M, Hofmann M, Johnson F, Kasprzak P, Kuhn K-H, Radtke U, Ratusinski C-M, RückertRI,RuppertV,SchulteS, Straeten G, Teßarek J, Umscheid T, Wack C (2008) Weiterbildung in endovaskulären Techniken mit der privaten Akademie der DGG. Gefasschirurgie 13:273-277

2. Schulte K-L, Amendt K, Hoffmann U, Tiefenbacher C, Weiss T, Mudra H, Hardung D, Nikol S (2012) Curriculum Interventionelle Therapie der arteriellen Gefäßerkrankungen. Kardiologe 6:375-389 
Hier steht eine Anzeige.

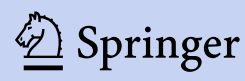

